\title{
Turbulence generator using a precessing sphere
}

$\operatorname{AUTHOR}(S):$

Goto, S; Ishii, N; Kida, S; Nishioka, M

CITATION:

Goto, S ... [et al]. Turbulence generator using a precessing sphere. PHYSICS OF FLUIDS 2007, 19(6): 061705.

ISSUE DATE:

2007-06

URL:

http://hdl.handle.net/2433/50095

\section{RIGHT:}

Copyright 2007 American Institute of Physics. This article may be downloaded for personal use only. Any other use requires prior permission of the author and the American Institute of Physics. 


\title{
Turbulence generator using a precessing sphere
}

\author{
Susumu Goto, Nobukazu Ishii, Shigeo Kida, and Michio Nishioka \\ Department of Mechanical Engineering and Science, Kyoto University, Yoshida-Honmachi, Sakyo, \\ Kyoto 606-8501, Japan
}

(Received 24 February 2007; accepted 3 May 2007; published online 28 June 2007)

\begin{abstract}
We propose a precessing sphere as a tabletop turbulence generator, which has less uncertainty in the setting of control parameters and the resulting high flow-reproducibility. The precession is realized by rotating the spin axis of a sphere around another axis (the precession axis). In our experiments, the two axes are fixed at right angles. The flow inside the sphere is governed only by two nondimensional parameters, one being Re (the Reynolds number defined by the maximum peripheral velocity around the spin axis) and the other being $\Gamma$ (the rate of precession). The range of parameters for sustaining turbulence is revealed by the time-series analysis of velocity fields measured by particle image velocimetry. Well-developed turbulence can be sustained even for $\Gamma$ of the order of a few percent when Re is beyond a few thousands. () 2007 American Institute of Physics. [DOI: 10.1063/1.2746040]
\end{abstract}

Turbulence has been a central problem for years in the field of fluid mechanics, and many types of turbulence generators have been proposed. In experimental studies of turbulence it is highly desirable to minimize the uncertainty of external conditions, since flow structures may be sensitive to the boundary conditions, which are generally complicated. Therefore, here we aim at proposing a precisely controllable mechanism to sustain developed turbulence inside a simple boundary. For this purpose, we use a precessing sphere (Fig. 1). Fluid is confined in a sphere of radius $a$, and it spins around an axis (the spin axis) at a constant angular velocity $\Omega_{s}$. This spinning sphere is further rotated around another axis (the precession axis) at a constant angular velocity $\Omega_{p}$. Here, we restrict ourselves to the case that the two axes are at right angles.

Flow structures inside a precessing cavity such as a sphere/spheroid, a spherical/spheroidal shell and a cylinder have been studied by many authors ${ }^{1-11}$ mainly from the geophysical viewpoint; e.g., investigating the origin of the magnetic field of the Earth, the liquid core of which is confined in the precessing spheroidal cavity of mantle. In the context of geophysics, the case that the precession rate (the Poincaré number) $\Gamma \equiv \Omega_{p} / \Omega_{s}$ is as small as $O\left(10^{-7}\right)$ is the main target. Nevertheless, the fact reported in the literature that turbulence is sustained even for small $\Gamma$ is encouraging from an engineering viewpoint as well. Since the parameter region for sustaining turbulence in a precessing sphere and the statistical property of the sustained turbulence are largely unknown, this article aims at unveiling the parameter range to realize developed turbulence.

As will be shown below in detail, although the driving mechanism is simple, local and global flow structures inside the sphere have a diversity. The most important feature of this system is that if and only if we control the two angular velocities of the rotations, there is no uncertainty in the external parameters apart from the kinematic viscosity of fluid. In our experiment, we precisely control the two angular velocities using pulse motors. The ranges of parameters are
$\Omega_{s} /(2 \pi)=0.1-0.6 \mathrm{~Hz}$ and $\Omega_{p} /(2 \pi)=0.002-1.2 \mathrm{~Hz}$. Thanks to this precise control of two angular velocities, we have confirmed high reproducibility of the flow. This is a great advantage of the current system. We use an acrylic sphere of radius $a=50 \mathrm{~mm}$, which is filled with water (degassed by a vacuum pump and ultrasonics). The temperature of water is monitored to determine the kinematic viscosity.

Before reporting results, we summarize the control parameters of the present system. The reciprocal $\Omega_{s}^{-1}$ of the spin angular velocity and the radius $a$ of sphere are adopted as the characteristic time and length scales, respectively. The equation of continuity, the Navier-Stokes equation, and the boundary condition are respectively expressed, in the precession frame that rotates with $\Omega_{p}$ around the precession axis, as

$$
\begin{aligned}
& \boldsymbol{\nabla} \cdot \boldsymbol{u}=0, \\
& \frac{\partial}{\partial t} \boldsymbol{u}+\boldsymbol{u} \cdot \boldsymbol{\nabla} \boldsymbol{u}=-\nabla p+\frac{\nu}{a^{2} \Omega_{s}} \nabla^{2} \boldsymbol{u}+2 \frac{\Omega_{p}}{\Omega_{s}} \boldsymbol{u} \times \hat{\boldsymbol{e}}_{p}, \\
& \boldsymbol{u}=\hat{\boldsymbol{e}}_{s} \times \boldsymbol{x} \quad(\text { on }|\boldsymbol{x}|=1)
\end{aligned}
$$

in terms of nondimensional variables. Here, $\boldsymbol{x}, \boldsymbol{u}(\boldsymbol{x}, t)$, and $p(\boldsymbol{x}, t)$ are the nondimensional position vector (with the origin at the center of the sphere), velocity, and pressure (including the centrifugal potential) fields, respectively. The third term on the right-hand side of (2) represents the Coriolis force. The constant unit vectors $\hat{\boldsymbol{e}}_{s}$ and $\hat{\boldsymbol{e}}_{p}$ are in the direction of the spin and precession axes, respectively. Since (1)-(3) depend only on the Reynolds number $\operatorname{Re} \equiv a^{2} \Omega_{s} / \nu$ and the precession rate $\Gamma$, these two serve as the control parameters.

It may be worth emphasizing, in passing, that we can carry out direct numerical simulations (DNS) for exactly same parameters as our laboratory experiment because the boundary condition (3) is precisely implemented in DNS.

The method of the measurement is as follows. We employ particle image velocimetry (PIV) to measure the velocity field inside the sphere. Small particles (Kanomax 


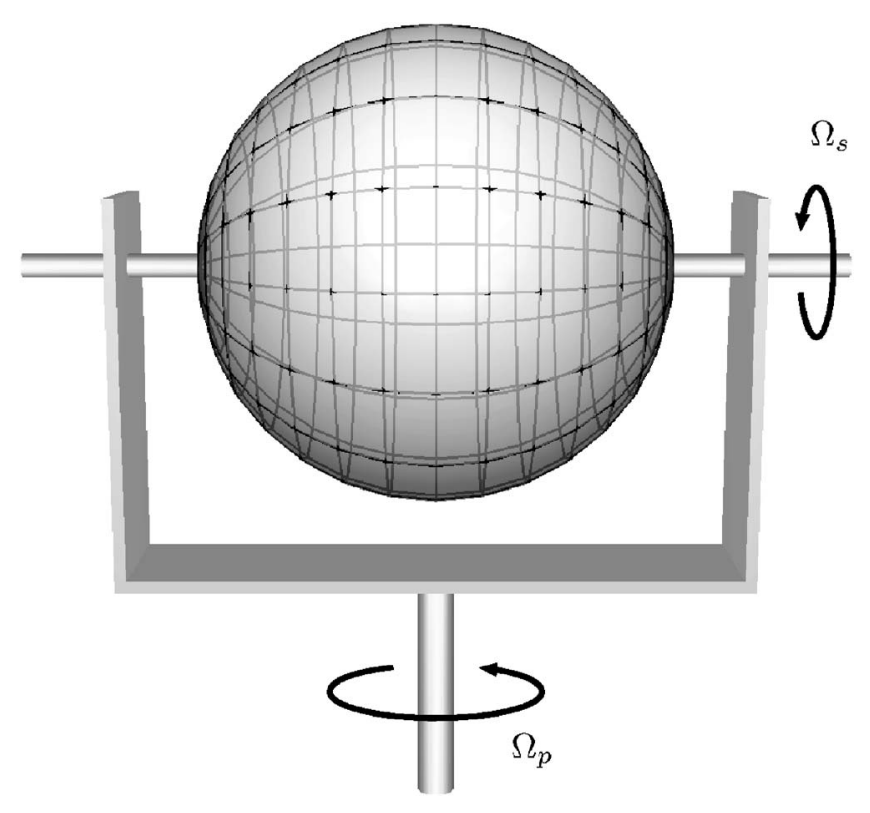

FIG. 1. Schematic picture of a precessing sphere.

ORGASOL; mean mass density and radius are $1.03 \mathrm{~g} / \mathrm{cm}^{3}$ and $50 \mu \mathrm{m}$, respectively.) are used as tracers. A laser sheet and a video camera (frame rate is set at a value between 10 and 30 frames per second depending on the flow velocity) are fixed in the precession frame; i.e., the frame in which (2) is described. The laser sheet is approximately perpendicular to the spin axis, and it is about $17 \mathrm{~mm}$ behind the center of the sphere. The thickness of the sheet in the measurement region is about $1 \mathrm{~mm}$. The video camera focuses on particles in a hemisphere with a view almost perpendicular to the laser sheet. By applying a direct cross-correlation method to the images thus recorded during the period over several times of the spin period $T_{s}\left(\equiv 2 \pi / \Omega_{s}\right)$, we estimate the velocity field on the laser sheet.

Measurements are carried out in a (statistically) steady state; i.e., after the duration of $O\left(100 T_{s}\right)$ from the instant when $\Omega_{p}$ and/or $\Omega_{s}$ are changed.

By the flow visualizations for $700 \leqq \operatorname{Re} \leqslant 30000$, it is found that for a fixed Re, the flow structures undergo drastic changes with $\Gamma$, and these changes are qualitatively independent of $\operatorname{Re}$ for $3000 \leqslant \operatorname{Re} \leqslant 30000$. Hence, we describe the results (Fig. 2) in detail for a fixed Re at about 4700 with changing $\Gamma$ as $0,0.01,0.04,0.1$, and 1 . The first column of Fig. 2 shows visualized tracers (the size of the shown region is about $40 \mathrm{~mm}$ width by $24 \mathrm{~mm}$ height) on the plane of measurement, and the second column shows the schematic picture of the global mean flow structure in the precession frame. As seen in these first two columns of Fig. 2, drastic changes in flow structures take place when $\Gamma$ increases from 0 to 1 even for a fixed Re. (a) When $\Gamma=0$, the fluid rotates like a solid body around the spin axis. Note that it is analytically shown by the energy equation that the flow inside a sphere rotating around a single axis with a constant angular velocity eventually tends to a solid-body rotation. (b) When $\Gamma$ is small, e.g., $\Gamma=0.01$, the global flow still shows a circulation around an axis nearly parallel to the spin axis, and the center of the swirl is shifted to the left in this figure com- pared to (a). The axis of the swirling flow inclines retrogradely around the precession axis due to the Coriolis effect. Since the laser sheet is located behind the center of the sphere, this tilt of the swirling flow is observed as the leftward shift in this figure. The tilt angle around the precession axis for this parameter is measured to be about $0.27 \mathrm{rad}$, which is in good agreement with the prediction $0.24 \mathrm{rad}$ by Busse's formula. $^{2}$ (c) As $\Gamma$ increases, the tilt of the swirling flow gets larger, and the flow experiences a Hopf bifurcation around $\Gamma=0.04$. (d) When $\Gamma$ further increases, the flow becomes turbulent. Here, we call the flows turbulent that are neither steady nor periodic. (e) However, if we further increases $\Gamma$ up to about 1 , the flow inside the sphere relaminarizes. It is interesting to observe that this laminar flow is accompanied with a global swirling motion around the precession axis. This swirling motion is observed as a rightward flow on this plane of measurement. Recall that the laser sheet plane is behind the center of the sphere. The rightward velocity in this frame of reference implies that the flow inside the sphere is swirling faster than the wall of the sphere. As expected, if we further increase $\Gamma$ to $O(10)$, the velocity in this frame almost vanishes (figure is omitted), which implies that the flow in the laboratory frame tends to the solidbody rotation around the precession axis as $\Gamma \rightarrow \infty$.

In order to judge whether the flow inside the sphere is regarded as turbulent or not, we employ a time-series analysis based on the PIV measurement. We measure the twodimensional velocity field $\boldsymbol{u}(\boldsymbol{x}, t)$ on the laser sheet (shown in the third column of Fig. 2) for the duration $0 \leq t \leq T$. Here, $u_{1}$ and $u_{2}$ are, respectively, the horizontal and vertical components of the measured velocity. In order to classify the flow into steady, periodic and turbulent flows, we estimate the two-time velocity correlation function,

$$
C_{i}(\boldsymbol{x}, \tau)=\frac{\left\langle\left[u_{i}(\boldsymbol{x}, t)-\mu_{i}(\boldsymbol{x})\right]\left[u_{i}(\boldsymbol{x}, t+\tau)-\mu_{i}(\boldsymbol{x})\right]\right\rangle}{\sigma_{i}^{2}(\boldsymbol{x})}
$$

for $i=1,2$. Here, brackets $\langle\cdot\rangle$ denote the temporal average, and $\mu_{i}(\boldsymbol{x})$ and $\sigma_{i}(\boldsymbol{x})$ are, respectively, the mean and standard deviation of $u_{i}$. The correlation function $C_{i}(\boldsymbol{x}, \tau)$ is estimated for the time interval $0 \leq \tau \leq T / 2$. We take the spatial (over the region shown in Fig. 2) and ensemble (over 20 realizations for $\Gamma=0,0.01,0.04$, and $1 ; 100$ realizations for $\Gamma=0.1)$ averages of $C_{i}(\boldsymbol{x}, \tau)$. The averaged correlation function $\bar{C}_{i}(\tau)$ is plotted in the fourth column of Fig. 2, where time interval $\tau$ is normalized by $T_{s}$.

Assuming the measured velocity is the sum of the signal and noise, the correlation function may be expressed as

$$
C_{i}(\tau)=\frac{{\widetilde{\sigma_{i}}}^{2}}{\tilde{\sigma}_{i}^{2}+\sigma_{i}^{\prime 2}} \widetilde{C}_{i}(\tau)+\frac{\sigma_{i}^{\prime 2}}{\tilde{\sigma}_{i}^{2}+\sigma_{i}^{\prime 2}} C_{i}^{\prime}(\tau),
$$

where $\widetilde{C}_{i}$ (or $C_{i}^{\prime}$ ) and $\tilde{\sigma}_{i}$ (or $\sigma_{i}^{\prime}$ ) are the two-time correlation function and standard deviation of the signal (or noise), respectively. Here, we have assumed that the noise is statistically independent of the signal.

By using (5), we can classify the flow based on $\bar{C}_{i}(\tau)$ as follows. (i) The correlation functions observed in Fig. 2(a) and 2(b) can be regarded as the ones for steady flow, since in 

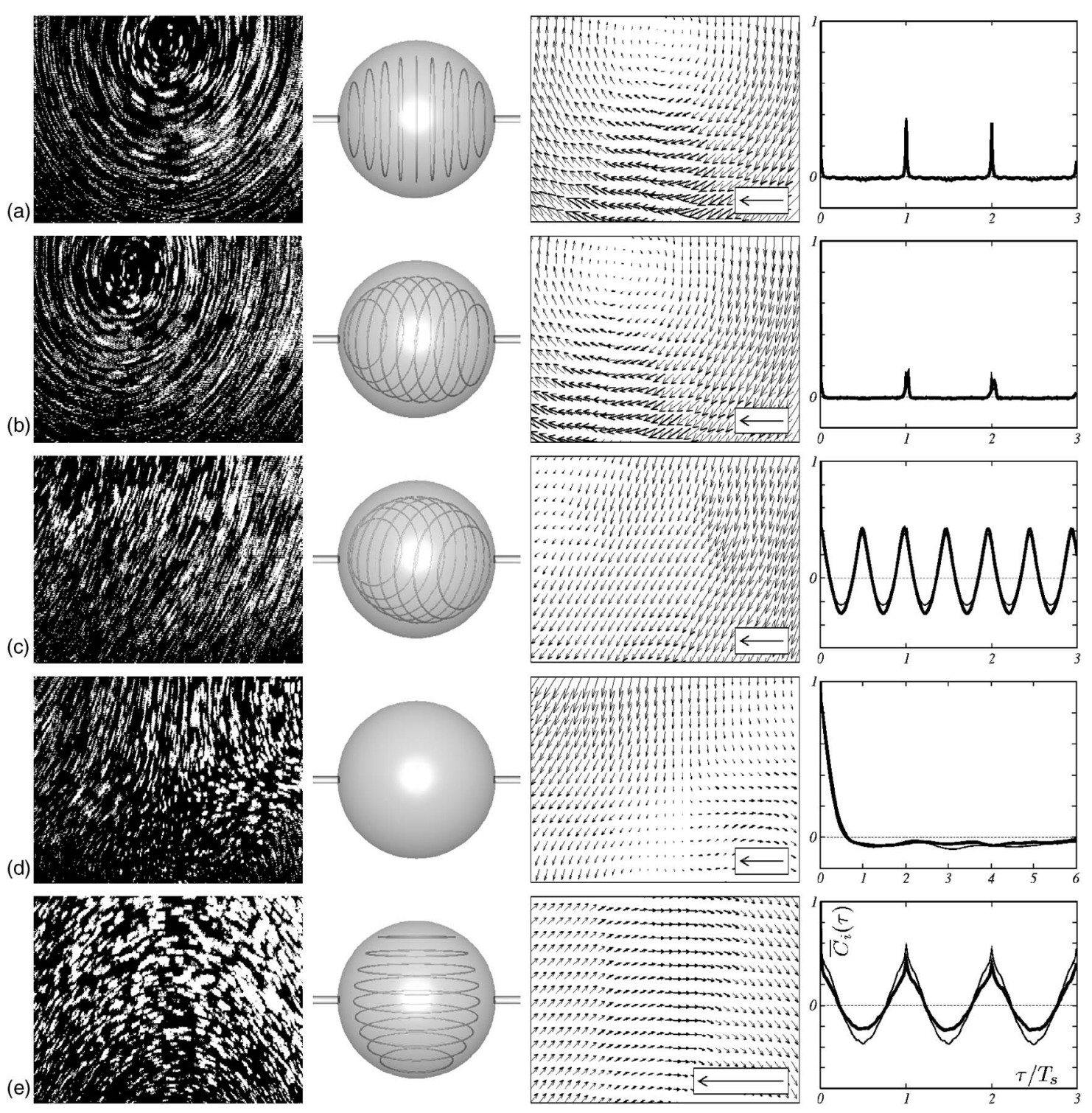

FIG. 2. Experimental results for a fixed Reynolds number $R e \approx 4700$ and (a) $\Gamma=0$, (b) 0.01 , (c) 0.04 , (d) 0.1 , and (e) 1 . Measurements are carried out in the precession frame. First column, visualized flow. Second column, schematic picture of mean flow (the viewpoint is perpendicular to the two axes). Third column, velocity field by PIV. The bottom right arrow indicates half the maximum wall velocity. The vector arrows plotted in (e) are magnified twice compared to (a-d). Fourth column, two-time correlation function of horizontal (thick curves) and vertical (thin curves) velocity components.

such a flow, $\tilde{\sigma_{i}}=0$. It is interesting to observe that the noise is almost white in time (because $C_{i}^{\prime}(\tau)$ has a sharp peak in the vicinity of the origin) but also has periodic components whose period coincides with the spin period $T_{s}$. This is reasonable because the camera periodically (with the period $T_{s}$ ) records images at exactly the same position. Note that the sphericity of the sphere is not necessarily exact, and that the perspective of the camera and the direction of the laser sheet change periodically due to the refraction. (ii) The correlation function observed in Fig. 2(c) implies that the flow is periodic in time. The Fourier transform of $\bar{C}_{i}(\tau)$ indicates that the period of the flow is about $0.48 T_{s}$ in this case, which is consistent with our preliminary DNS result. (iii) The correlation function observed in Fig. 2(d) is not regarded as the one for steady or periodic flows. Thus, we classify this flow as turbulent. It is seen that the correlation function rapidly decays in $\tau<T_{s}$, and remains small negative values for the duration of the order of the precession period $\left(2 \pi / \Omega_{p}\right.$ $=10 T_{s}$ in this case). (iv) The correlation function in Fig. 2(e) is periodic, and we classify this flow as periodic. It is interesting to observe that the period is exactly same as $T_{s}$.

By analyzing the correlation function for various combinations of Re and $\Gamma$, we show in Fig. 3 the parameter sets where turbulence is maintained. It is of importance to observe in Fig. 3 that when Re is larger than about 3000, turbulence is always maintained by setting $\Gamma$ appropriately. The range of $\Gamma$ where turbulence is sustained gets wider as $\operatorname{Re}$ increases. It might be impressive that turbulence is maintained even with very small $\Gamma$ of $O(0.01)$ for larger Re. This implies that as far as the magnitude $|\boldsymbol{\Omega}|\left(=\sqrt{\Omega_{s}^{2}+\Omega_{p}^{2}} \approx \Omega_{s}\right)$ of the total angular velocity of the sphere is sufficiently large, its unstationarity $|\boldsymbol{\Omega}| /|\boldsymbol{\Omega}|\left(=\Omega_{s} \Omega_{p} / \sqrt{\Omega_{s}^{2}+\Omega_{p}^{2}} \approx \Omega_{p}\right)$ can be only $1 \%$ of $\Omega_{s}$ for generating turbulence. This feature of the precessing sphere is in contrast to the fact that the flow inside 


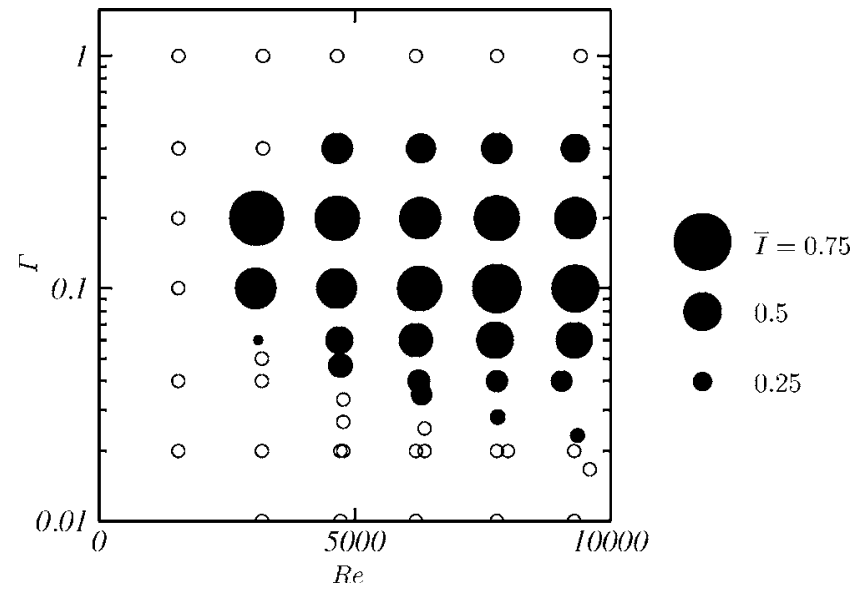

FIG. 3. Parameter sets $(\bullet)$ to realize sustained turbulence. The radius of symbols is proportional to the mean intensity. Open circles denote the parameters for laminar flows.

a sphere rotating around a single axis tends to be a solidbody rotation irrespective of the Reynolds number.

It is also interesting that even if $\mathrm{Re}$ is fixed, we can change the degree of turbulence by tuning the magnitude of $\Gamma$. To show this, we define the degree of turbulence at each point by the ratio of the rms value of velocity fluctuation to that of the total velocity,

$$
I(\boldsymbol{x})=\sqrt{\left\langle|\boldsymbol{u}(\boldsymbol{x}, t)-\langle\boldsymbol{u}(\boldsymbol{x})\rangle|^{2}\right\rangle} / \sqrt{\left\langle|\boldsymbol{u}(\boldsymbol{x})|^{2}\right\rangle} .
$$

Spatial and ensemble averaged values $\bar{I}$ of $I(\boldsymbol{x})$ are indicated by the size of symbols in Fig. 3. It is seen in this figure that the degree of turbulence is maximized at a relatively small $\Gamma$ (say, around 0.1) for each Reynolds number. In such a case, the turbulence seems almost without a mean flow in the frame of the measurement. For smaller (or larger) $\Gamma$ than this value, turbulence tends to be accompanied with a global circulation around the spin (or precession) axis.

The above is the first report of our experiment based on the measurements by a relatively low-speed camera. Flow structures in high-speed regions (e.g., in the boundary layer) or at high Reynolds numbers are near-future targets. Also open are the transition mechanism to turbulence, the critical parameters, the detailed phase diagram, the existence/ nonexistence of hysteresis, ${ }^{1}$ and the statistical features of turbulence sustained (such as spatial correlation functions, energy spectra, a turbulence intensity, and their Reynoldsnumber dependence, which are important in comparing our turbulence generator with other ones). Instability properties are under investigation by the help of DNS and will be reported elsewhere.

In summary, using a precessing sphere, we propose a compact turbulence generator. One important feature of this generator is that the control of external parameters is easy and precise. This fact encourages us to use this generator as a standard laboratory simulator of turbulence for comparing various characteristics of turbulence (mixing, transport, etc.) among researchers in different laboratories. Another important feature is that we can easily realize well-developed turbulence inside the sphere. Typically, at $\operatorname{Re}=O\left(10^{4}\right)$, welldeveloped turbulence is sustained when $\Gamma=\Omega_{p} / \Omega_{s}$ is only the order of 0.1. Incidentally, a precessing spheroid, rather than a sphere, probably produces developed turbulence more efficiently due to pressure effect in addition to the viscous force on the wall. With these results we may propose the present method of turbulence generation as, for example, a new type of mixer without stirrers.

This study is supported by the 21st Century COE Program on Complex Functional Mechanical Systems, and Grant-in-Aid for Exploratory Research from the Ministry of Education, Culture, Sports, Science and Technology. The authors thank Professor Satoshi Sakai for his kind advice when building up the apparatus.

${ }^{1}$ W. V. R. Malkus, "Precession of the earth as the cause of geomagnetism," Science 160, 259 (1968).

${ }^{2}$ F. H. Busse, "Steady fluid flow in a precessing spheroidal shell," J. Fluid Mech. 33, 739 (1968).

${ }^{3} \mathrm{R}$. Manasseh, "Breakdown regimes of inertia waves in a precessing cylinder," J. Fluid Mech. 243, 261 (1992).

${ }^{4}$ J. Vanyo, P. Wilde, P. Cardin, and P. Olson, "Experiments on precessing flows in the Earth's liquid core," Geophys. J. Int. 121, 136 (1995).

${ }^{5}$ R. Hollerbach and R. R. Kerswell, "Oscillatory internal shear layers in rotating and precessing flows," J. Fluid Mech. 298, 327 (1995).

${ }^{6}$ J. J. Kobine, "Inertial wave dynamics in a rotating and precessing cylinder," J. Fluid Mech. 303, 233 (1995).

${ }^{7}$ J. P. Vanyo and J. R. Dunn, "Core precession: flow structures and energy," Geophys. J. Int. 142, 409 (2000).

${ }^{8}$ A. Tilgner and F. H. Busse, "Fluid flows in precessing spherical shells," J. Fluid Mech. 426, 387 (2001).

${ }^{9}$ S. Lorenzani and A. Tilgner, "Fluid instabilities in precessing spheroidal cavities," J. Fluid Mech. 447, 111 (2001).

${ }^{10}$ J. Noir, D. Jault, and P. Cardin, "Numerical study of the motions within a slowly precessing sphere at low Ekman number," J. Fluid Mech. 437, 282 (2001).

${ }^{11}$ J. Noir, P. Cardin, D. Jault, and J.-P. Masson, "Experimental evidence of nonlinear resonance effects between retrograde precession and the tilt-over mode within a spheroid," Geophys. J. Int. 154, 407 (2003). 